Supplemental Information for Computational Studies

\title{
Unsaturated Cyclopentadienyl-Molybdenum and Tungsten Carbonyl Cluster Complexes Containing Pd- and $\operatorname{Pt}\left(\mathrm{PBu}_{3}^{\mathrm{t}}\right)$ Groups
}

\author{
Richard D. Adams*, Jack L. Smith, Jr., and Carl B. Hollandsworth \\ Department of Chemistry and Biochemistry, University of South Carolina, Columbia, SC, 29208
}

Details Computational Treatments. Starting coordinates for the title compounds were obtained from X-ray crystal structure data. Geometry optimizations and single-point energy calculations were performed using the Amsterdam Density Functional (ADF) program, version 2004.01, developed by Baerends, et al. ${ }^{9}$ Geometry optimizations were carried out, with $C_{2}$ symmetry restraints, using the local exchange-correlation potential of Vosko et al. ${ }^{10}$ and the non-local exchange and correlation corrections of Perdew and Wang (PW91). ${ }^{11}$ For the $\mathrm{Pt}$ and $\mathrm{W}$ atoms, the electronic configuration was described using a double- $\zeta$ Slater-type orbital (STO) basis for $5 \mathrm{~s}$ and triple- $\zeta$ orbitals for $6 \mathrm{~s}, 5 \mathrm{p}, 5 \mathrm{~d}$, and $4 \mathrm{f}$, augmented by $6 \mathrm{p}$ and $5 \mathrm{f}$ polarization functions. The core was frozen and defined to be $4 \mathrm{~d}$ and below. For the Mo and Pd atoms, the electronic configuration was described using a double- $\zeta$ STO basis for $4 \mathrm{~s}$ and triple- $\zeta$ for $5 \mathrm{~s}, 4 \mathrm{p}$, and $4 \mathrm{~d}$ augmented by a $5 \mathrm{p}$ polarization function. The core was frozen and defined to be $3 \mathrm{~d}$ and below. For the phosphorus atoms, a triple- $\zeta$ STO basis was used for $3 \mathrm{~s}$ and $3 \mathrm{p}$. This basis was augmented with $3 \mathrm{~d}$ and $4 \mathrm{f}$ polarization functions while the core was frozen at $2 \mathrm{p}$ and below. For oxygen and carbon, double- $\zeta$ basis sets were employed for $2 \mathrm{~s}$ and $2 \mathrm{p}$, augmented with $3 \mathrm{~d}$ and $4 \mathrm{f}$ polarization functions, and the 1 s core was frozen. A double- $\zeta$ basis was used for the hydrogen atoms augmented by $2 \mathrm{p}$ and $3 \mathrm{~d}$ polarization functions. 
Corrections for relativistic effects were employed in each calculation by using the Zeroth Order Regular Approximation (ZORA) method. ${ }^{12}$ Accuracy and convergence parameters were left at the default level with the exception of the general integration accuracy, which was set to 7.0 .

Results

I. Converged Cartesian Coordinates $(\AA)$ from DFT Geometry Optimizations

a. $\mathrm{Cp}_{2} \mathrm{Mo}_{2} \mathrm{Pt}_{2}\left(\mu_{2}-\mathrm{CO}\right)_{4}\left(\mathrm{Bu}_{3}^{\mathrm{t}} \mathrm{P}\right)_{2}, 9$, Computation Started with Pt-Pt distance $=3.10 \AA$

b. $\mathrm{Cp}_{2} \mathrm{Mo}_{2} \mathrm{Pt}_{2}\left(\mu_{2}-\mathrm{CO}\right)_{4}\left(\mathrm{Bu}_{3}^{\mathrm{t}} \mathrm{P}\right)_{2}, 9$, Computation Started with Pt-Pt distance $=3.50 \AA$

c. $\mathrm{Cp}_{2} \mathrm{Mo}_{2} \mathrm{Pd}_{2}\left(\mu_{2}-\mathrm{CO}\right)_{4}\left(\mathrm{Bu}_{3}^{\mathrm{t}} \mathrm{P}\right)_{2}, \mathbf{1 0}$

d. $\mathrm{Cp}_{2} \mathrm{~W}_{2} \mathrm{Pt}_{2}\left(\mu_{2}-\mathrm{CO}\right)_{4}\left(\mathrm{Bu}_{3}^{\mathrm{t}} \mathrm{P}\right)_{2}, 11$

e. $\mathrm{Cp}_{2} \mathrm{~W}_{2} \mathrm{Pd}_{2}\left(\mu_{2}-\mathrm{CO}\right)_{4}\left(\mathrm{Bu}_{3}^{\mathrm{t}} \mathrm{P}_{2}, \mathbf{1 2}\right.$

f. $\left[\mathrm{Cp}_{2} \mathrm{Mo}_{2} \mathrm{Pd}_{2}\left(\mu_{2}-\mathrm{CO}\right)_{4}\left(\mathrm{Bu}_{3}^{\mathrm{t}} \mathrm{P}\right)_{2}\right]^{2-},[\mathbf{1 0}]^{2-}$

II. Selected Bond Distances ( $\AA$ ) for DFT Optimized Geometries for 9-12 and [10] $]^{2-}$. 


\title{
I. Converged Cartesian Coordinates $(\AA)$ from DFT Geometry Optimizations
}

\author{
a. $\mathrm{Cp}_{2} \mathrm{Mo}_{2} \mathrm{Pt}_{2}\left(\mu_{2}-\mathrm{CO}\right)_{4}\left(\mathrm{Bu}_{3}^{\mathrm{t}} \mathrm{P}\right)_{2}, 9$, Computation Started with Pt-Pt distance $=3.10 \AA$
}

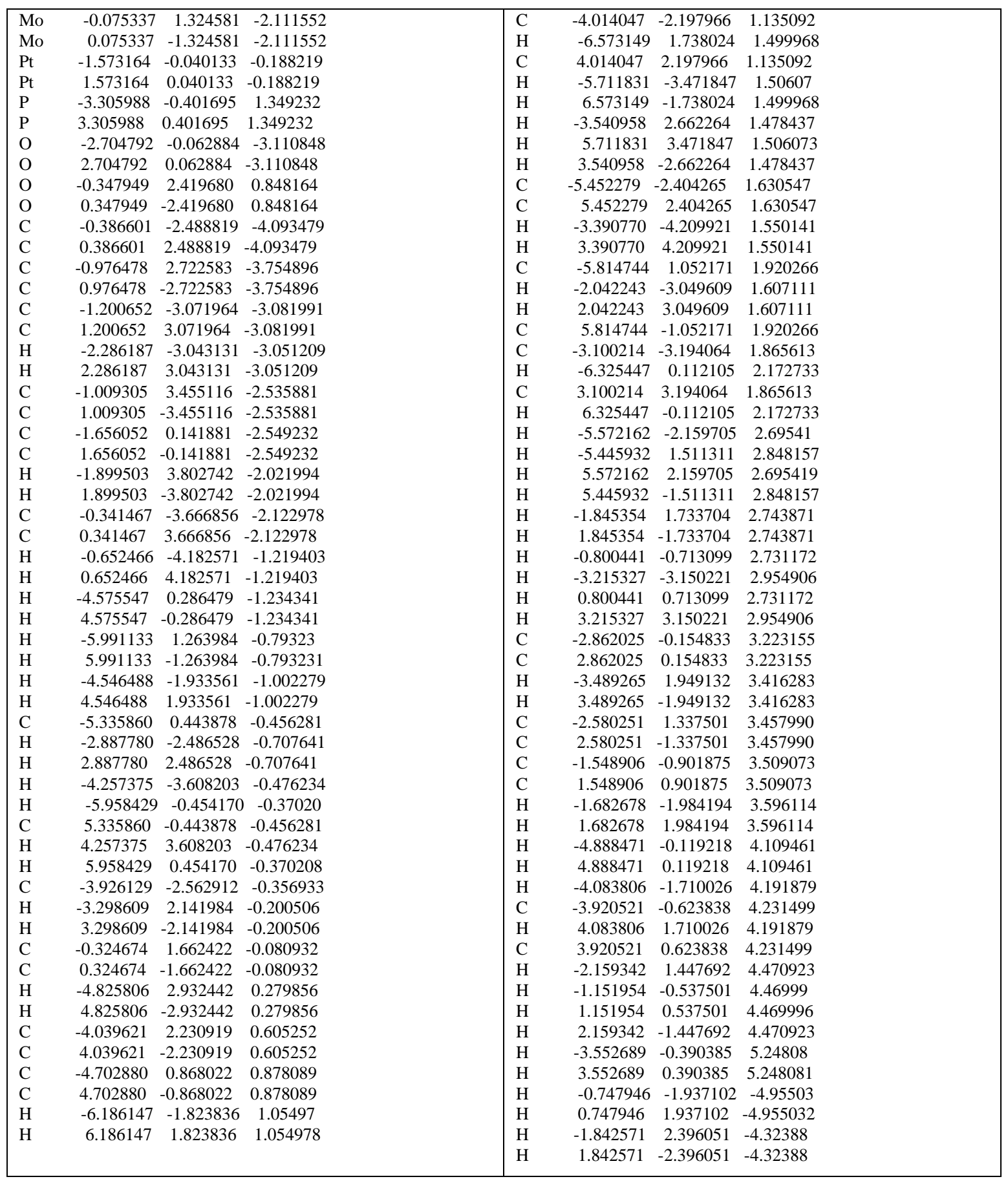




\section{b. $\mathrm{Cp}_{2} \mathrm{Mo}_{2} \mathrm{Pt}_{2}\left(\mu_{2}-\mathrm{CO}\right)_{4}\left(\mathrm{Bu}_{3}^{\mathrm{t}} \mathrm{P}\right)_{2}, 9$, Computation Started with Pt-Pt distance $=3.50 \AA$}

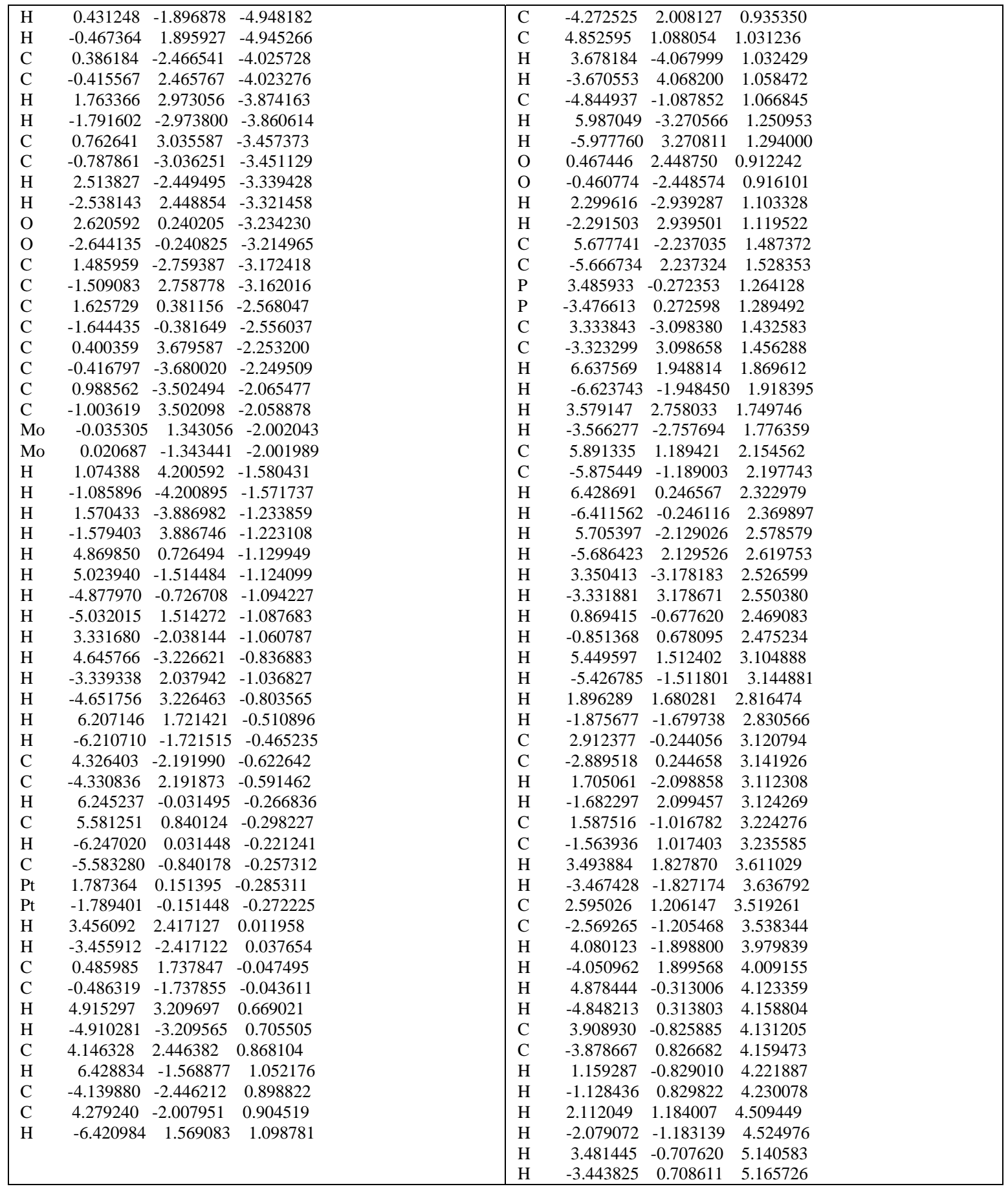




\section{c. $\mathrm{Cp}_{2} \mathrm{Mo}_{2} \mathrm{Pd}_{2}\left(\mu_{2}-\mathrm{CO}\right)_{4}\left(\mathrm{Bu}_{3}^{\mathrm{t}} \mathrm{P}\right)_{2}, \mathbf{1 0}$}

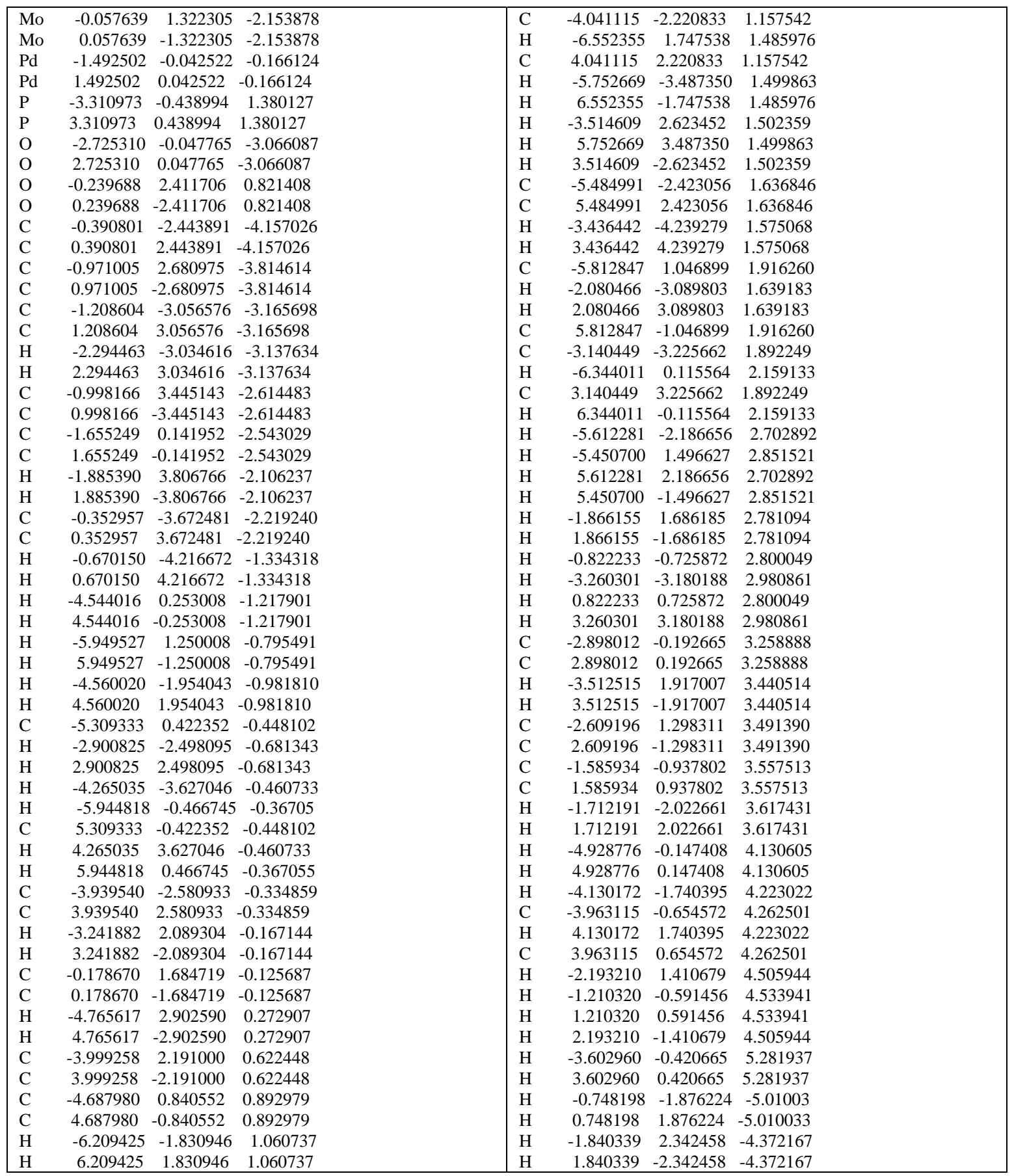




\section{d. $\mathrm{Cp}_{2} \mathrm{~W}_{2} \mathrm{Pt}_{2}\left(\mu_{2}-\mathrm{CO}\right)_{4}\left(\mathrm{Bu}_{3}^{\mathrm{t}} \mathrm{P}\right)_{2}, \mathbf{1 1}$}

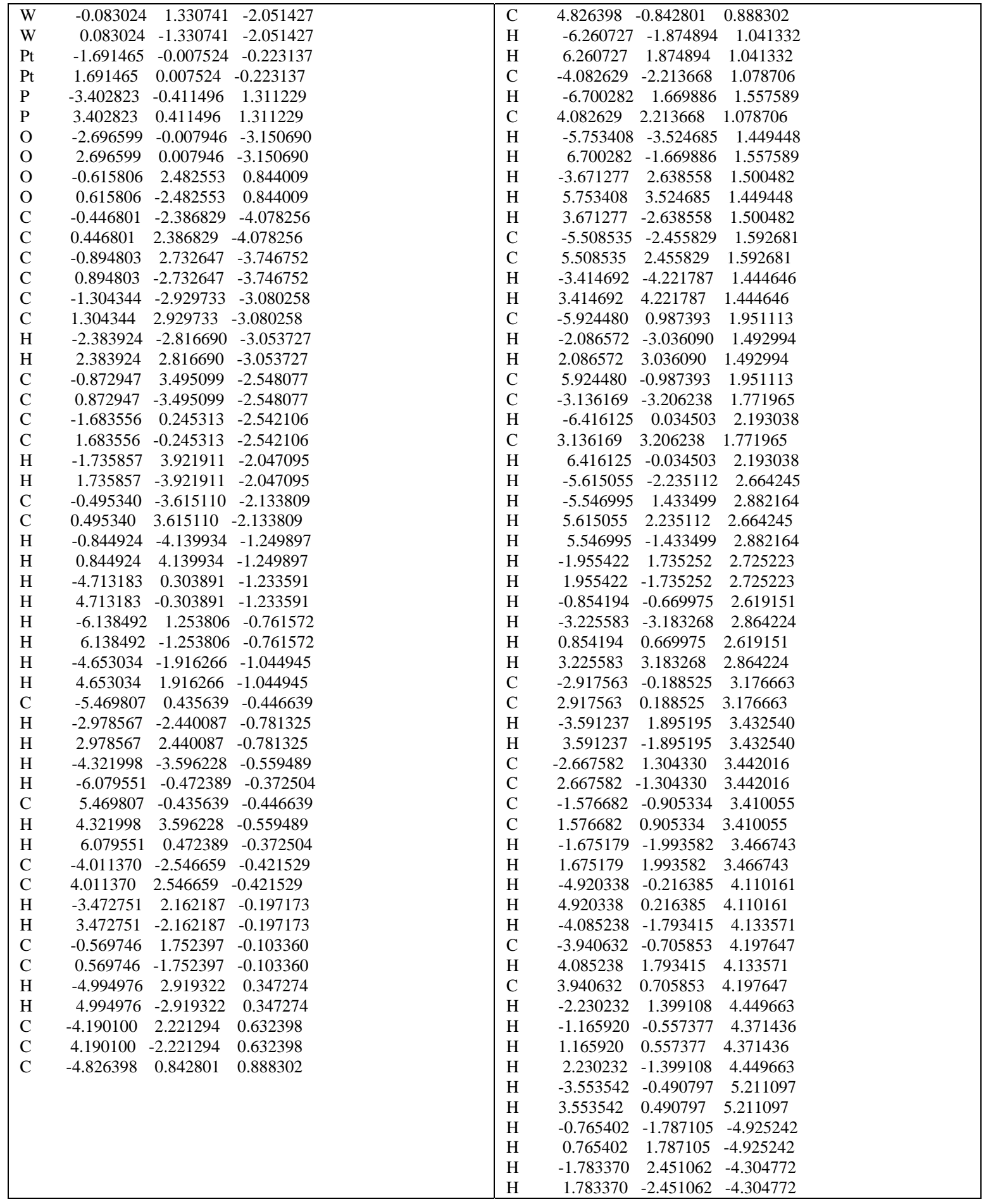




\section{e. $\mathrm{Cp}_{2} \mathrm{~W}_{2} \mathrm{Pd}_{2}\left(\mu_{2}-\mathrm{CO}\right)_{4}\left(\mathrm{Bu}_{3}^{\mathrm{t}} \mathrm{P}\right)_{2}, 12$}

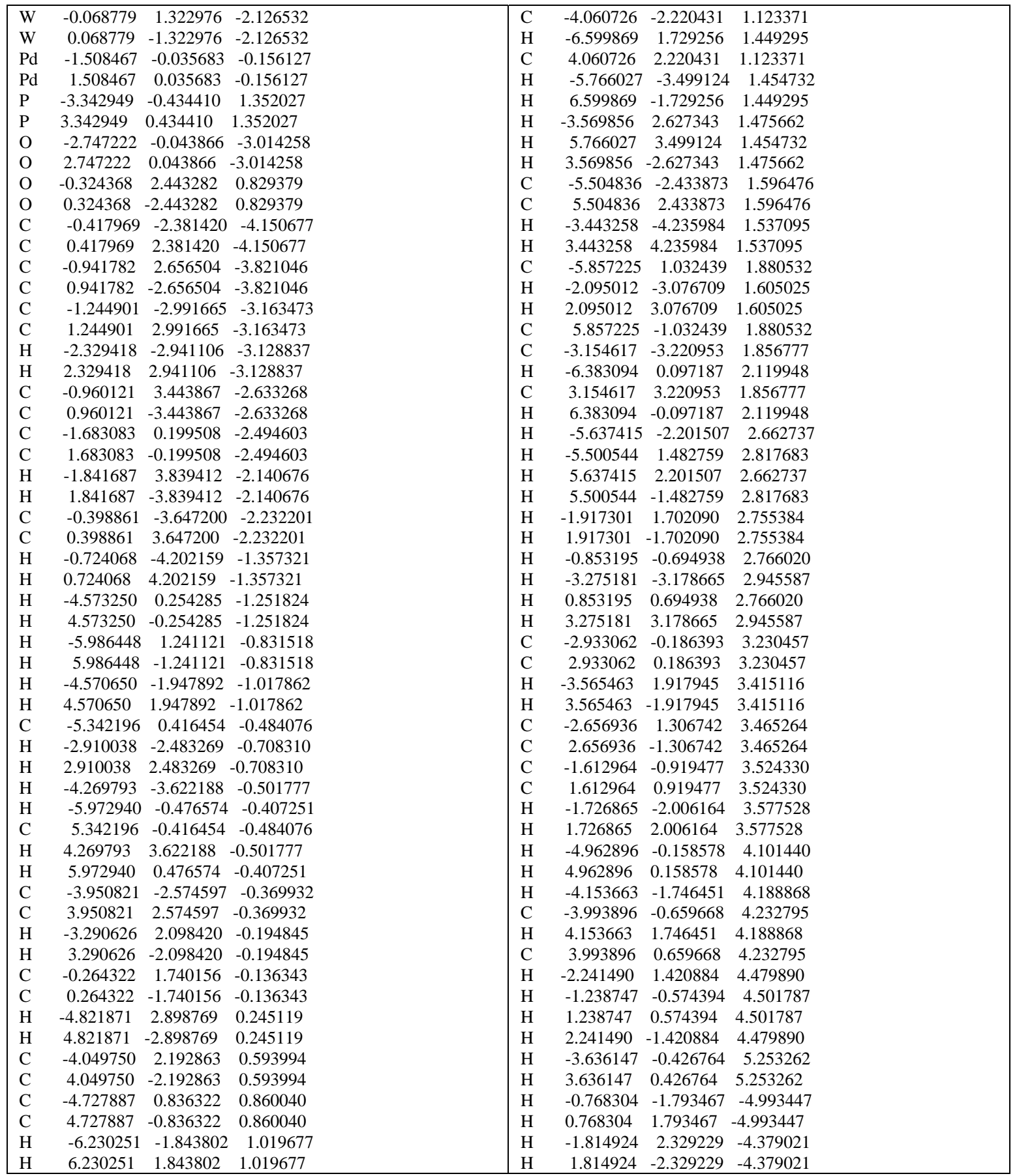




\section{f. $\left[\mathrm{Cp}_{2} \mathrm{Mo}_{2} \mathrm{Pd}_{2}\left(\mu_{2}-\mathrm{CO}\right)_{4}\left(\mathrm{Bu}_{3}^{\mathrm{t}} \mathrm{P}\right)_{2}\right]^{2-},[\mathbf{1 0}]^{2-}$}

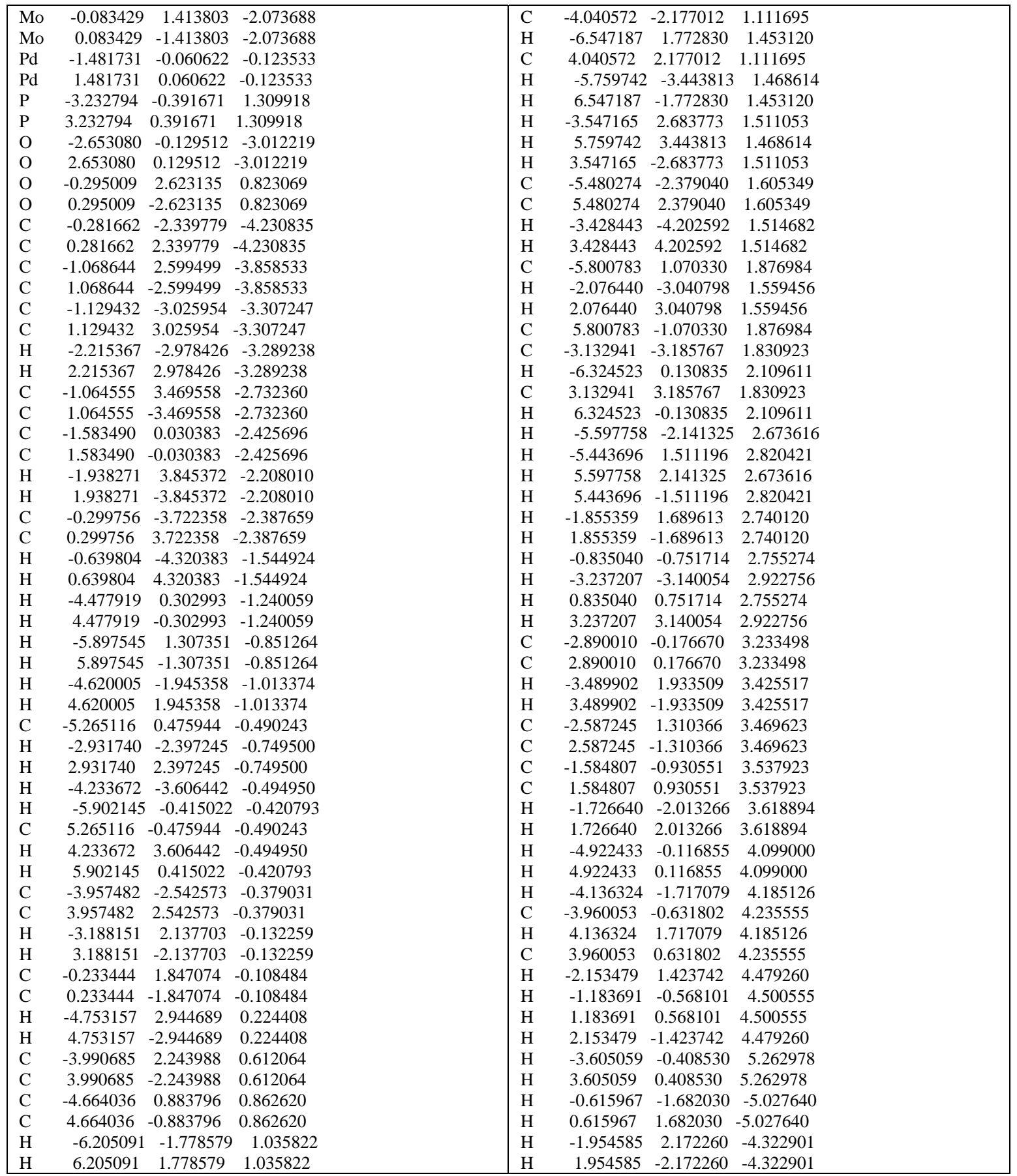


II. Selected Bond Distances ( $)$ ) for DFT Optimized Geometries for 9-12 and [10] ${ }^{2-}$.

\begin{tabular}{|c|c|c|c|c|c|c|}
\hline Bond & $\begin{array}{c}9 \text { (Pt-Pt start } \\
=3.10 \AA)\end{array}$ & $\begin{array}{c}9 \text { (Pt-Pt start } \\
=3.50 \AA)\end{array}$ & 10 & {$[10]^{2-}$} & 11 & 12 \\
\hline $\mathrm{M}-\mathrm{M}^{\mathrm{a}}$ & 2.653 & 2.687 & 2.647 & 2.833 & 2.667 & 2.650 \\
\hline M-M' 1 & 2.794 & 2.773 & 2.806 & 2.816 & 2.779 & 2.793 \\
\hline M-M' 2 & 2.840 & 2.881 & 2.827 & 2.843 & 2.871 & 2.833 \\
\hline $\mathrm{M}^{\prime}-\mathrm{M}^{\prime}$ & 3.147 & 3.599 & 2.986 & 2.966 & 3.383 & 3.018 \\
\hline M'-P & 2.345 & 2.338 & 2.420 & 2.287 & 2.334 & 2.408 \\
\hline $\mathrm{M}-\mathrm{C}(\mathrm{O}) 1^{\mathrm{b}}$ & 2.022 & 2.001 & 2.024 & 2.071 & 1.995 & 2.001 \\
\hline $\mathrm{M}-\mathrm{C}(\mathrm{O}) 2^{\mathrm{c}}$ & 2.311 & 2.423 & 2.287 & 2.233 & 2.418 & 2.350 \\
\hline $\mathrm{M}-\mathrm{C}(\mathrm{O}) 3^{\mathrm{d}}$ & & 2.061 & 2.064 & 2.018 & 2.052 & 2.043 \\
\hline $\mathrm{M}^{\prime}-\mathrm{C}(\mathrm{O}) 1^{\mathrm{e}}$ & 2.114 & 2.066 & 2.171 & 2.280 & 2.090 & 2.168 \\
\hline$M^{\prime}-C(O) 2^{f}$ & 2.499 & 2.966 & 2.343 & 2.477 & 2.859 & 2.459 \\
\hline C-O 1 (M-M) & 1.207 & 1.206 & 1.206 & 1.230 & 1.209 & 1.209 \\
\hline $\mathrm{C}-\mathrm{O} 2\left(\mathrm{M}^{\prime}-\mathrm{M}^{\prime}\right)$ & 1.199 & 1.194 & 1.196 & 1.214 & 1.197 & 1.196 \\
\hline
\end{tabular}

a. $\mathrm{M}=\mathrm{Mo}$ or $\mathrm{W}$ and $\mathrm{M}^{\prime}=\mathrm{Pd}$ or $\mathrm{Pt}$

b. $\mathrm{M}-\mathrm{C}(\mathrm{O}) 1$ is the short distance between $\mathrm{Mo}$ or $\mathrm{W}$ and the $\mathrm{CO}$ ligand carbon atom that bridges the Mo-Mo or W-W vector.

c. $\mathrm{M}-\mathrm{C}(\mathrm{O}) 2$ is the long distance between $\mathrm{Mo}$ or $\mathrm{W}$ and the $\mathrm{CO}$ ligand carbon atom that bridges the Mo-Mo or W-W vector.

d. $\mathrm{M}-\mathrm{C}(\mathrm{O}) 3$ is the distance between $\mathrm{Mo}$ or $\mathrm{W}$ and the carbon atom for the $\mathrm{CO}$ ligand that bridges the $\mathrm{Mo} / \mathrm{W}$ to $\mathrm{Pd} / \mathrm{Pt}$ vector.

e. $\mathrm{M}^{\prime}-\mathrm{C}(\mathrm{O}) 1$ is the short distance between $\mathrm{Pt}$ or $\mathrm{Pd}$ and the carbon atom for the $\mathrm{CO}$ ligand that bridges the Pt-Pt or Pd-Pd vector.

f. $\mathrm{M}^{\prime}-\mathrm{C}(\mathrm{O}) 2$ is the long distance between $\mathrm{Pt}$ or $\mathrm{Pd}$ and the carbon atom for the $\mathrm{CO}$ ligand that bridges the $\mathrm{Pt}-\mathrm{Pt}$ or $\mathrm{Pd}-\mathrm{Pd}$ vector. 